\title{
Nitric Oxide Inhibitory Constituents from the Fruits of Amomum tsao-ko
}

\author{
Jun Gu Kim, Thi Phuong Linh Le, Hye Ryeong Hong, Jae Sang Han, Jun Hwi Ko, \\ Seung Hyun Lee, Mi Kyeong Lee, and Bang Yeon Hwang* \\ College of Pharmacy, Chungbuk National University, Cheongju 28160, Republic of Korea
}

\begin{abstract}
Bioactivity-guided fractionation of $\mathrm{MeOH}$ extract of the dried fruits of Amomum tsao-ko led to isolation of nine compounds (1 - 9). Their structures were elucidated by spectroscopic methods including extensive $1 \mathrm{D}$ and 2D-NMR, as alpinetin (1), naringenin-5- $O$-methyl ether (2), naringenin (3), hesperetin (4), 2', $4^{\prime}, 6^{\prime}-$ trihydroxy-4-methoxy chalcone (5), tsaokoin (6), boesenbergin B (7), 4-hydroxyboesenbergin B (8), and tsaokoarylone (9). Of these, compound $\mathbf{8}$ was isolated from a natural source for the first time, which was previously reported as a synthetic product. The isolated compounds $(\mathbf{1}-\mathbf{9})$ were tested for their inhibitory effects on LPS-induced nitric oxide production in RAW 264.7 macrophages. Among them, three chalcone derivatives (compounds 5, 7, and 8) and a diarylheptanoid (compound 9) exhibited significant inhibitory activity on the NO production with $\mathrm{IC}_{50}$ values ranging from 10.9 to $22.5 \mu \mathrm{M}$.
\end{abstract}

Keywords - Amomum tsao-ko, Zingiberaceae, Nitric oxide, Anti-inflammatory activity

\section{Introduction}

Amoтum tsao-ko Crevost et Lemaire (Zingiberaceae) is a medicinal plant that is widely used in Korea, China, and Japan, for the treatment of nausea, abdominal distension, diarrhea, and malaria. ${ }^{1}$ Previous phytochemical investigations on this plant reported the presence of monoterpenes, diarylheptanoids, flavonoids, and phenolic compounds. ${ }^{2-5}$ In the course of a research program for the isolation of anti-inflammatory constituents from medicinal plants, a $\mathrm{MeOH}$ extract of the fruits of $A$. tsao-ko showed significant inhibitory effects on LPS-induced nitric oxide (NO) production $\left(\mathrm{IC}_{50}\right.$ value of $23.5 \mu \mathrm{g} / \mathrm{mL}$ ). Subsequently, the fruits of $A$. tsao-ko were extracted with $\mathrm{MeOH}$, and the extract was suspended in water and then partitioned with $n$-hexane, $\mathrm{CH}_{2} \mathrm{Cl}_{2}$, EtOAc, and $n$-BuOH. Bioassayguided fractionation of the $\mathrm{CH}_{2} \mathrm{Cl}_{2}$ - and ethyl acetatesoluble fraction led to the isolation of 9 known compounds (1-9). The structures of isolates were determined by interpretation on their 1D and 2D NMR spectroscopic data as well as mass spectrometry, and by comparison of the spectroscopic data with literature. The isolated compounds (1 - 9) were tested for their inhibitory effects on LPS-induced nitric oxide production in RAW

\footnotetext{
*Author for correspondence

Bang Yeon Hwang, College of Pharmacy, Chungbuk National University, Cheongju 28160, Republic of Korea

Tel: +82-43-261-2814; E-mail: byhwang@chungbuk.ac.kr
}

264.7 macrophages. Herein, the isolation, structural determination, and the nitric oxide inhibitory effects of the isolated compounds are reported.

\section{Experimental}

General experimental procedures - Optical rotations were measured on a JASCO DIP-1000 polarimeter. UV spectra were recorded on a JASCO UV-550 spectrophotometer, and IR spectra were measured on a JASCO FT-IR 4100 spectrometer. NMR spectra were recorded on a Bruker AVANCE 400 and $500 \mathrm{MHz}$ spectrometer. ESIMS and HRESIMS were obtained on LCQ Fleet and maXis 4G mass spectrometers, respectively. Column chromatography was performed on silica gel (Merck, 70 230 and 230 - 400 mesh) and Lichroprep RP-18 (Merck, $40-63 \mu \mathrm{m})$. MPLC was performed on a Biotage Isolera Prime chromatography system. Preparative HPLC was performed using Waters HPLC system equipped with two Waters 515 pumps with a 2996 photodiode-array detector using a YMC J'sphere ODS-H80 $(4 \mu \mathrm{m}, 150 \times 20 \mathrm{~mm}$, i.d., flow rate $6 \mathrm{~mL} / \mathrm{min}$ ). TLC was performed using precoated silica gel $60 \mathrm{~F}_{254}(0.25 \mathrm{~mm}$, Merck) plates, and spots were detected by a $10 \%$ vanillin- $\mathrm{H}_{2} \mathrm{SO}_{4}$ in water spray reagent.

Plant material - The dried fruits of A. tsao-ko $(3 \mathrm{~kg})$ were purchased from Kyungdong herbal market in Seoul, Korea, in May 2014. A voucher specimen (CBNU-2014- 
05-AT) was authenticated by B. Y. Hwang and deposited at the Herbarium of the College of Pharmacy, Chungbuk National University, Korea.

Extraction and isolation - The dried fruits of $A$. tsao$k o(3 \mathrm{~kg})$ were extracted with $\mathrm{MeOH}(3 \times 5 \mathrm{~L})$ at room temperature and filtered. The filtrates were evaporated under reduced pressure to give a $\mathrm{MeOH}$ extract (132 g), which was suspended in water and then successively partitioned with $n$-hexane, $\mathrm{CH}_{2} \mathrm{Cl}_{2}$, EtOAc, and $n$-BuOH. The $\mathrm{CH}_{2} \mathrm{Cl}_{2}$-soluble fraction (92 g) was chromatographed on a silica gel column (70 - 230 mesh), eluting with a gradient solvent system consisting of $n$-hexane $/ \mathrm{CH}_{2} \mathrm{Cl}_{2} /$ $\mathrm{MeOH}$ (4:1:0 to 0:1:1) yielded 8 fractions (ATC1 - ATC8). Fraction ATC $6(31.9 \mathrm{~g})$ was further separated on a silica gel column (230-400 mesh) with a gradient solvent system of $\mathrm{CH}_{2} \mathrm{Cl}_{2} / \mathrm{MeOH}$ (40:1 to $1: 1$ ) as the eluent to give six sub-fractions (ATC6-1 - ATC6-6). Fraction ATC 6-2 (2.7 g) was purified by preparative HPLC (MeCN$\mathrm{H}_{2} \mathrm{O}, 30: 70$ to $\left.60: 40\right)$ to afford compound $1(1.7 \mathrm{mg})$. Fraction ATC 6-4 (2.2 g) was further purified by preparative HPLC (MeCN-H $\mathrm{H}_{2} \mathrm{O}, 20: 80$ to 50:50) to afford compound 2 (4.2 mg). The EtOAc-soluble fraction (21 g) was chromatographed on a silica gel column (230 - 400 mesh) and eluted with $n$-hexane/EtOAc gradient system (20:1 to $1: 2$ ) to afford 10 fractions (ATE1 - ATE10). Fraction ATE 2 (324 mg) was separated by MPLC with Lichroprep RP-18 column with $\mathrm{MeOH}-\mathrm{H}_{2} \mathrm{O}$ gradient system (30:70 to 100:0) to give compound 7 (14.0 mg). Fraction ATE 7 (1.7 g) was separated on a Sephadex LH20 column and eluted with $\mathrm{MeOH}-\mathrm{H}_{2} \mathrm{O}(80: 20)$ to give compound $8(16.0 \mathrm{mg})$. Fraction ATE $8(2.2 \mathrm{~g})$ was further separated by MPLC with Lichroprep RP-18 column and eluted with $\mathrm{MeOH}-\mathrm{H}_{2} \mathrm{O}$ (5:95 to 100:0) to give 12 fractions (ATE 8-1 - ATE 8-12). Fraction ATE 8-5 was purified by preparative HPLC $\left(\mathrm{MeCN}-\mathrm{H}_{2} \mathrm{O}, 35: 65\right.$, isocratic) to afford compound $\mathbf{6}(13.0 \mathrm{mg})$. Fraction ATE 8-7 was further purified by preparative $\mathrm{HPLC}\left(\mathrm{MeCN}-\mathrm{H}_{2} \mathrm{O}\right.$, $35: 65$, isocratic) to afford compounds $3(1.2 \mathrm{mg})$ and 4 $(0.8 \mathrm{mg})$. Fraction ATE $8-8$ was further separated by preparative HPLC $\left(\mathrm{MeCN}-\mathrm{H}_{2} \mathrm{O}, 40: 60\right.$ to $\left.45: 55\right)$ to afford compounds $5(2.2 \mathrm{mg})$ and $9(1.5 \mathrm{mg})$.

Alpinetin (1) - Colorless needles, $[\alpha]_{\mathrm{D}}{ }^{25}:-3.1 \quad(c=0.1$ $\mathrm{MeOH})$; UV (MeOH) $\lambda_{\max } \mathrm{nm}: 209,286,320$; IR (KBr) $v_{\max } \mathrm{cm}^{-1}: 3520,3200,3075,1636,1610,1580,1474$, 1315, 1274, 1110; ESIMS $m / z: 271.17[\mathrm{M}+\mathrm{H}]^{+}$; ${ }^{1} \mathrm{H}$ NMR (DMSO- $\left.d_{6}, 500 \mathrm{MHz}\right): \delta 7.36 \sim 7.5\left(5 \mathrm{H}, \mathrm{m}, \mathrm{H}-2^{\prime}, 3^{\prime}, 4^{\prime}, 5^{\prime}\right.$, 6'), $6.07(1 \mathrm{H}, \mathrm{d}, J=2.0 \mathrm{~Hz}, \mathrm{H}-6), 6.00(1 \mathrm{H}, \mathrm{d}, J=2.0 \mathrm{~Hz}$, H-8), 5.5 (1H, dd, $J=3.0,12.5 \mathrm{~Hz}, \mathrm{H}-2), 3.74(3 \mathrm{H}, \mathrm{s}$, $\left.\mathrm{OCH}_{3}\right), 3.01(1 \mathrm{H}, \mathrm{dd}, J=12.5,16.5 \mathrm{~Hz}, \mathrm{H}-3 \alpha), 2.65(1 \mathrm{H}$, $\mathrm{dd}, J=3.0,16.5 \mathrm{~Hz}, \mathrm{H}-3 \beta) ;{ }^{13} \mathrm{C}$ NMR (DMSO- $d_{6}, 125$
MHz): $\delta 187.7$ (C-4), 165.1 (C-7), 164.5 (C-9), 162.6 (C5), 139.7 (C-1'), 129.0 (C-3', 5'), 128.8 (C-4'), 126.9 (C-2', 6'), 104.8 (C-10), 96.2 (C-6), 93.9 (C-8), 78.4 (C-2), 56.1 $\left(\mathrm{OCH}_{3}\right), 45.3(\mathrm{C}-3)$.

Naringenin-5- $\boldsymbol{O}$-methyl ether (2)-Colorless oil, $[\alpha]_{\mathrm{D}}{ }^{25}$ : $-14.0\left(c=0.01, \mathrm{CHCl}_{3}\right) ; \mathrm{UV}(\mathrm{MeOH}) \lambda_{\max } \mathrm{nm}$ : 226, 283; IR (KBr) $v_{\max } \mathrm{cm}^{-1}: 3366,1642$; ESIMS $\mathrm{m} / \mathrm{z}$ : $287.17[\mathrm{M}+\mathrm{H}]^{+} ;{ }^{1} \mathrm{H}$ NMR (DMSO- $d_{6}, 400 \mathrm{MHz}$ ): $\delta 7.3$ $\left(2 \mathrm{H}, \mathrm{d}, J=8.8 \mathrm{~Hz}, \mathrm{H}-2^{\prime}, 6^{\prime}\right), 6.79$ (2H, d, $J=8.8 \mathrm{~Hz}, \mathrm{H}-3$ ', $\left.5^{\prime}\right), 6.04(1 \mathrm{H}, \mathrm{d}, J=2.0 \mathrm{~Hz}, \mathrm{H}-6), 5.93(1 \mathrm{H}, \mathrm{d}, J=2.0 \mathrm{~Hz}$, H-8), 5.34 (1H, dd, $J=2.8,12.4 \mathrm{~Hz}, \mathrm{H}-2), 3.73$ (3H, s, $\left.\mathrm{OCH}_{3}\right), 2.98(1 \mathrm{H}, \mathrm{dd}, J=12.4,16.3 \mathrm{~Hz}, \mathrm{H}-3 \alpha), 2.48(1 \mathrm{H}$, $\mathrm{dd}, J=3.1,16.4 \mathrm{~Hz}, \mathrm{H}-3 \beta) ;{ }^{13} \mathrm{C}$ NMR (DMSO- $d_{6}, 100$ $\mathrm{MHz}): \delta 188.2$ (C-4), 164.9 (C-5), 164.7 (C-7), 162.7 (C9), 158.0 (C-4'), 131.0 (C-1'), 128.6 (C-2', 6'), 115.6 (C-3', 5'), 104.9 (C-10), 96.1 (C-6), 93.7 (C-8), 78.5 (C-2), 56.1 $\left(\mathrm{OCH}_{3}\right), 45.2$ (C-3).

Naringenin (3) - Colorless gum, $[\alpha]_{\mathrm{D}}^{25}:-17.5(c=0.1$, $\mathrm{MeOH})$; UV (MeOH) $\lambda_{\max } \mathrm{nm}: 248,293$; IR $(\mathrm{KBr}) v_{\max }$ $\mathrm{cm}^{-1}$ : 3112, 1600, 1517, 1493, 1459, 1246, 1178, 1154; ESIMS $m / z: 273.10[\mathrm{M}+\mathrm{H}]^{+} ;{ }^{1} \mathrm{H}$ NMR $\left(\mathrm{CD}_{3} \mathrm{OD}, 500\right.$ $\mathrm{MHz}): \delta 7.32\left(2 \mathrm{H}, \mathrm{d}, J=8.6 \mathrm{~Hz}, \mathrm{H}-2^{\prime}, 6^{\prime}\right), 6.82(2 \mathrm{H}, \mathrm{d}$, $\left.J=8.6 \mathrm{~Hz}, \mathrm{H}-3 ', 5^{\prime}\right), 5.89$ (1H, d, $\left.J=2.1 \mathrm{~Hz}, \mathrm{H}-6\right), 5.88$ $(1 \mathrm{H}, \mathrm{d}, J=2.1 \mathrm{~Hz}, \mathrm{H}-8), 5.36(1 \mathrm{H}, \mathrm{dd}, J=3.0,13.0 \mathrm{~Hz}$, $\mathrm{H}-2), 3.14$ (1H, dd, $J=13.0,17.0 \mathrm{~Hz}, \mathrm{H}-3 \alpha), 2.71(1 \mathrm{H}$, $\mathrm{dd}, J=3.0,17.0 \mathrm{~Hz}, \mathrm{H}-3 \beta) ;{ }^{13} \mathrm{C} \mathrm{NMR}\left(\mathrm{CD}_{3} \mathrm{OD}, 125\right.$ $\mathrm{MHz}): \delta 197.8$ (C-4), 168.6 (C-7), 165.5 (C-5), 164.9 (C9), 159.1 (C-4'), 131.1 (C-1'), 129.0 (C-2', 6'), 116.3 (C-3', 5'), 103.3 (C-10), 97.1 (C-6), 96.2 (C-8), 80.5 (C-2), 44.1 (C-3).

Hesperetin (4) - Colorless gum, $[\alpha]_{\mathrm{D}}^{25}:-10.6(c=0.1$, $\mathrm{MeOH})$; UV (MeOH) $\lambda_{\max } \mathrm{nm}: 288,325$; IR (KBr) $v_{\text {max }}$ $\mathrm{cm}^{-1}: 3499,3433,1635,1586,1514,1473,1442$; ESIMS $m / z: 303.1[\mathrm{M}+\mathrm{H}]^{+} ;{ }^{1} \mathrm{H}$ NMR $\left(\mathrm{CD}_{3} \mathrm{OD}, 500 \mathrm{MHz}\right): \delta 7.08$ $(1 \mathrm{H}, \mathrm{d}, J=2.0 \mathrm{~Hz}, \mathrm{H}-2 '), 6.93$ (1H, dd, $J=2.0,8.2 \mathrm{~Hz}, \mathrm{H}-$ $\left.6^{\prime}\right), 6.82(1 \mathrm{H}, \mathrm{d}, J=8.2 \mathrm{~Hz}, \mathrm{H}-5 '), 5.91(1 \mathrm{H}, \mathrm{d}, J=2.1 \mathrm{~Hz}$, H-6), 5.88 (1H, d, $J=2.1 \mathrm{~Hz}, \mathrm{H}-8), 5.36(1 \mathrm{H}, \mathrm{dd}, J=3.0$, $13.0 \mathrm{~Hz}, \mathrm{H}-2), 3.88\left(3 \mathrm{H}, \mathrm{s}, \mathrm{OCH}_{3}\right), 3.17(1 \mathrm{H}, \mathrm{dd}, J=13.0$, $17.0 \mathrm{~Hz}, \mathrm{H}-3 \alpha), 2.73$ (1H, dd, $J=3.0,17.0 \mathrm{~Hz}, \mathrm{H}-3 \beta)$; ${ }^{13} \mathrm{C}$ NMR $\left(\mathrm{CD}_{3} \mathrm{OD}, 125 \mathrm{MHz}\right): \delta 197.8(\mathrm{C}-4), 167.2(\mathrm{C}-$ 5), 165.5 (C-7), 164.9 (C-9), 149.2 (C-3'), 148.2 (C-4'), 131.8 (C-1'), 120.6 (C-2'), 116.2 (C-5'), 111.3 (C-6'), 103.4 (C-10), 97.2 (C-6), 96.3 (C-8), 80.5 (C-2), 56.5 $\left(\mathrm{OCH}_{3}\right), 44.2(\mathrm{C}-3)$.

2',4',6'-Trihydroxy-4-methoxy chalcone (5) - Colorless gum, UV (MeOH) $\lambda_{\max } \mathrm{nm}: 296,360$; IR (KBr) $v_{\max } \mathrm{cm}^{-1}$ : 3327, 1646; ESIMS $m / z$ : $287.0[\mathrm{M}+\mathrm{H}]^{+}$; ${ }^{1} \mathrm{H}$ NMR $\left(\mathrm{CD}_{3} \mathrm{OD}, 400 \mathrm{MHz}\right): \delta 7.8(1 \mathrm{H}, \mathrm{d}, J=15.5 \mathrm{~Hz}, \mathrm{H}-\alpha)$, $7.70(1 \mathrm{H}, \mathrm{d}, J=15.5 \mathrm{~Hz}, \mathrm{H}-\beta), 7.52(2 \mathrm{H}, \mathrm{d}, J=8.6 \mathrm{~Hz}$, H-2, 6), 6.84 (2H, d, J=8.6 Hz, H-3, 5), $6.01(1 \mathrm{H}, \mathrm{d}$, 
$\left.J=2.2 \mathrm{~Hz}, \mathrm{H}-3^{\prime}\right), 5.93\left(1 \mathrm{H}, \mathrm{d}, J=2.2 \mathrm{~Hz}, \mathrm{H}-5^{\prime}\right), 3.93(3 \mathrm{H}$, $\left.\mathrm{s}, \mathrm{OCH}_{3}\right) ;{ }^{13} \mathrm{C} \mathrm{NMR}\left(\mathrm{CD}_{3} \mathrm{OD}, 100 \mathrm{MHz}\right): \delta 194.0(\mathrm{C}=\mathrm{O})$, 168.6 (C-2'), 166.4 (C-4'), 164.7 (C-6'), 161.2 (C-4), 143.8 (C- $\beta$ ), 131.3 (C-3, 5), 130.3 (C-1), 128.4 (C- $\alpha$ ), 116.8 (C-2, 6), 106.6 (C-1'), 97.1 (C-3'), 92.4 (C-5'), 56.4 $\left(\mathrm{OCH}_{3}\right)$.

Tsaokoin (6) - Pale brown oil; UV (MeOH) $\lambda_{\max } \mathrm{nm}$; 228, 231; IR (KBr) $v_{\max } \mathrm{cm}^{-1}: 3396,2868,2728,1683$, 1634, 1428, 1379, 1181, 1062, 1026, 963, 903, 740; ESIMS $m / z: 167.08[\mathrm{M}+\mathrm{H}]^{+} ;{ }^{1} \mathrm{H}$ NMR $\left(\mathrm{CDCl}_{3}, 400\right.$ MHz): $\delta 9.41(1 \mathrm{H}, \mathrm{s}, \mathrm{H}-10), 6.66(1 \mathrm{H}, \mathrm{ddd}, J=5.6,2.8$, $1.2 \mathrm{~Hz}, \mathrm{H}-3), 4.07(1 \mathrm{H}, \mathrm{dt}, J=9.2,5.0 \mathrm{~Hz}, \mathrm{H}-5), 2.98(\mathrm{H}$, m, H-1), 2.48 (1H, dddt, $J=18.8,5.6,5.0,1.4 \mathrm{~Hz}, \mathrm{H}-4 \beta$ ), $2.42(1 \mathrm{H}, \mathrm{m}, \mathrm{H}-6), 2.38(1 \mathrm{H}, \mathrm{ddt}, J=18.8,9.2,2.8 \mathrm{~Hz}, \mathrm{H}-$ $4 \alpha), 2.02(1 \mathrm{H}, \mathrm{m}, \mathrm{H}-9 \beta), 1.73(1 \mathrm{H}, \mathrm{m}, \mathrm{H}-7 \alpha), 1.54(1 \mathrm{H}$, m, H-7ß), $1.50(2 \mathrm{H}, \mathrm{m}, \mathrm{H}-8) .1 .40(1 \mathrm{H}, \mathrm{m}, \mathrm{H}-9 \alpha) ;{ }^{13} \mathrm{C}$ NMR (CD 3 OD, $125 \mathrm{MHz}): \delta 194.5$ (C-10), 148.1 (C-3), 144.7 (C-2), 67.4 (C-5), 42.8 (C-6), 37.1 (C-1), 31.9 (C9), 30.3 (C-4), 24.5 (C-7), 24.2 (C-8).

Boesenbergin B (7) - Pale yellow amorphous solid, $[\alpha]_{\mathrm{D}}{ }^{25}:-12.1\left(c=1.10, \mathrm{CHCl}_{3}\right) ; \mathrm{UV}(\mathrm{MeOH}) \lambda_{\max } \mathrm{nm}$; 233, 304, 349; IR (KBr) $v_{\max } \mathrm{cm}^{-1}: 3500,1630,1450$, 1380, 1100, 900; ESIMS $m / z$ : $405.08[\mathrm{M}+\mathrm{H}]^{+}$; ${ }^{1} \mathrm{H}$ NMR $\left(\mathrm{CDCl}_{3}, 400 \mathrm{MHz}\right): \delta 14.5\left(1 \mathrm{H}, \mathrm{s}, 2^{\prime}-\mathrm{OH}\right), 7.90(1 \mathrm{H}, \mathrm{d}$, $J=15.6 \mathrm{~Hz}, \mathrm{H}-\alpha), 7.79(1 \mathrm{H}, \mathrm{d}, J=15.6 \mathrm{~Hz}, \mathrm{H}-\beta), 7.61$ (2H, m, H-2, 6), 7.41 (1H, m, H-4), 7.38 (2H, m, H-3, 5), $6.74\left(1 \mathrm{H}, \mathrm{d}, J=10.0 \mathrm{~Hz}, \mathrm{H}-1^{\prime \prime}\right), 5.91\left(1 \mathrm{H}, \mathrm{s}, \mathrm{H}-5^{\prime}\right), 5.42$ $\left(1 \mathrm{H}, \mathrm{d}, J=10.0 \mathrm{~Hz}, \mathrm{H}-2^{\prime \prime}\right), 5.12\left(1 \mathrm{H}, \mathrm{m}, \mathrm{H}-7^{\prime \prime}\right), 3.92(3 \mathrm{H}$, s, 6'- $\left.\mathrm{OCH}_{3}\right), 2.13$ (2H, m, H-6"), $1.81(2 \mathrm{H}, \mathrm{m}, \mathrm{H}-4 "), 1.66$ (3H, s, $\left.\mathrm{CH}_{3}-9^{\prime \prime}\right), 1.57\left(3 \mathrm{H}, \mathrm{s}, \mathrm{CH}_{3}-10^{\prime \prime}\right), 1.42\left(3 \mathrm{H}, \mathrm{s}, \mathrm{CH}_{3}-\right.$ $\left.5^{\prime \prime}\right) ;{ }^{13} \mathrm{C} \mathrm{NMR}\left(\mathrm{CDCl}_{3}, 100 \mathrm{MHz}\right): \delta 192.6(\mathrm{C}=\mathrm{O}), 162.6$ (C-6'), 162.5 (C-2'), 160.8 (C-4'), 142.1 (C- $\beta$ ), 135.6 (C1), 131.9 (C-8"), 130.0 (C-4), 128.9 (C-2, 6), 128.3 (C-3, 5), 127.6 (C- $\alpha), 124.2$ (C-2"), 123.8 (C-7"), 116.5 (C-1"), 105.9 (C-1'), 102.8 (C-3'), 91.3 (C-5'), 80.8 (C-3"), 55.9 $\left(6 '-\mathrm{OCH}_{3}\right), 41.7$ (C-4"), 27.2 (C-5"), 25.7 (C-9"), 22.7 (C6"), 17.7 (C-10").

4-Hydroxyboesenbergin B (8) - Yellowish oil, $[\alpha]_{D}^{25}$ : -6.9 ( $c=0.1, \mathrm{MeOH})$; UV $(\mathrm{MeOH}) \lambda_{\max } \mathrm{nm} ; 233,304$, 349; IR (KBr) $v_{\max } \mathrm{cm}^{-1}: 3497,1625,1430,1380,1100$; ESIMS $m / z$ : $421.17[\mathrm{M}+\mathrm{H}]^{+}$; HRESIMS $m / z$ : 421.2009 $[\mathrm{M}+\mathrm{H}]^{+}$(cacld for $\left.\mathrm{C}_{26} \mathrm{H}_{29} \mathrm{O}_{5}, 421.2010\right)$; ${ }^{1} \mathrm{H} \mathrm{NMR}\left(\mathrm{CDCl}_{3}\right.$, $400 \mathrm{MHz})$ and ${ }^{13} \mathrm{C} \mathrm{NMR}\left(\mathrm{CDCl}_{3}, 100 \mathrm{MHz}\right)$, see Table 1.

Tsaokoarylone (9) - Colorless oil, $[\alpha]_{\mathrm{D}}{ }^{25}:+8.3(c=0.12$, $\mathrm{MeOH})$; UV (MeOH) $\lambda_{\max } \mathrm{nm}$; 224, 279; IR (KBr) $v_{\max }$ $\mathrm{cm}^{-1}: 3355,1705,1614,1515,1365,1242$, 830; ESIMS $m / z: 325.14[\mathrm{M}+\mathrm{H}]^{+} ;{ }^{1} \mathrm{H}$ NMR $\left(\mathrm{CD}_{3} \mathrm{OD}, 500 \mathrm{MHz}\right): \delta$ $7.41(1 \mathrm{H}, \mathrm{dd}, J=15.4,10.6 \mathrm{~Hz}, \mathrm{H}-5), 7.13(1 \mathrm{H}, \mathrm{d}, J=2.0$ Hz, H-2"), 7.04 (2H, d, $J=8.6$ Hz, H-2', 6'), 7.01 (1H, dd, $J=8.4,2.0 \mathrm{~Hz}, \mathrm{H}-6 "), 6.98(1 \mathrm{H}, \mathrm{d}, J=15.4 \mathrm{~Hz}, \mathrm{H}-6)$, $6.88\left(1 \mathrm{H}, \mathrm{dd}, J=15.4,10.6 \mathrm{~Hz}, \mathrm{H}-4^{\prime}\right), 6.78(1 \mathrm{H}, \mathrm{d}, J=8.2$ Hz, H-5"), 6.69 (2H, d, J=8.6 Hz, H-3", 5"), $6.28(1 \mathrm{H}, \mathrm{d}$, $J=15.4 \mathrm{~Hz}, \mathrm{H}-4), 3.89\left(3 \mathrm{H}, \mathrm{s}, 3 "-\mathrm{OCH}_{3}\right), 2.91(2 \mathrm{H}, \mathrm{m}$, $\mathrm{H}-1), 2.84$ (2H, m, H-2); ${ }^{13} \mathrm{C}$ NMR $\left(\mathrm{CD}_{3} \mathrm{OD}, 125 \mathrm{MHz}\right)$ : б 202.7 (C-3), 156.6 (C-4'), 149.5 (C-4"), 149.3 (C-3"), 146.2 (C-5), 143.9 (C-7), 133.3 (C-1'), 130.4 (C-2', 6'), 129.8 (C-1"), 129.0 (C-4), 125.2 (C-6), 123.0 (C-6"), 116.5 (C-5"), 116.2 (C-3', 5'), 111.0 (C-2"), $56.4\left(3^{\prime \prime}-\mathrm{OCH}_{3}\right)$, 43.2 (C-2), 30.9 (C-1).

Measurement of LPS-induced NO production and cell viability - RAW 264.7 cells were seeded into 96-well culture plates at $2 \times 10^{6}$ cells $/ \mathrm{mL}$, and stimulated with 1 $\mu \mathrm{g} / \mathrm{mL}$ of LPS in the presence or absence of compounds. After incubation at $37^{\circ} \mathrm{C}$ for $24 \mathrm{~h}$, nitrite concentration in culture medium was measured with the Griess reagent [containing equal volumes of $2 \%(\mathrm{w} / \mathrm{v})$ sulfanilamide in $5 \%(\mathrm{w} / \mathrm{v})$ phosphoric acid and $0.2 \%(\mathrm{w} / \mathrm{v})$ of $\mathrm{N}-(1-$ naphthyl) ethylenediamine solution] as an indicator of $\mathrm{NO}$ production. The absorbance was measured at $550 \mathrm{~nm}$ against a calibration curve with sodium nitrite standards. Cell viability of the remaining cells was determined by MTT (Sigma Chemical Co., St. Louis, MO)-based colorimetric assay.

\section{Results and Discussion}

In the continuing search for the plant-derived inhibitors of nitric oxide (NO) production, repeated column chromatographic separation of $\mathrm{CH}_{2} \mathrm{Cl}_{2}$ and EtOAc-soluble fractions resulted in the isolation of nine compounds (1 - 9) (Fig. 1). Their structures were identified as alpinetin $(\mathbf{1}),{ }^{6}$ naringenin5-O-methyl ether (2), ${ }^{7}$ naringenin (3), ${ }^{8}$ hesperetin (4), ${ }^{9}$ $2^{\prime}, 4^{\prime}, 6$ '-trihydroxy-4-methoxy chalcone $(\mathbf{5}),{ }^{10}$ tsaokoin $(\mathbf{6}),{ }^{11}$ boesenbergin B (7), ${ }^{12}$ 4-hydroxyboesenbergin B (8), ${ }^{13}$ and tsaokoarylone (9). ${ }^{14}$ Among them, compound $\mathbf{8}$ was previously reported but only as a synthetic product. The full NMR assignments of $\mathbf{8}$ were determined by analysis of 2D NMR such as HMQC and HMBC spectroscopic data.

Compound $\mathbf{8}$ was obtained as yellowish oil. The molecular formula of $\mathbf{8}$ was determined to be $\mathrm{C}_{26} \mathrm{H}_{28} \mathrm{O}_{5}$ by the ${ }^{13} \mathrm{C}$ NMR data and an HRESIMS ion peak at $\mathrm{m} / \mathrm{z}$ $421.2009[\mathrm{M}+\mathrm{H}]^{+}$(calcd 421.2010), requiring thirteen indices of hydrogen deficiency. The ${ }^{1} \mathrm{H}$ NMR spectrum of 8 indicated the presence of a pair of characteristic trans olefinic protons at the $\mathrm{C}-\alpha$ and $\mathrm{C}-\beta$ positions of a chalcone skeleton $\left[\delta_{\mathrm{H}} 7.79(1 \mathrm{H}, \mathrm{d}, J=15.7 \mathrm{~Hz})\right.$ and $7.76(1 \mathrm{H}, \mathrm{d}$, $J=15.7 \mathrm{~Hz})$ ], and revealed presence of $p$-disubstituted benzene ring $\left[\delta_{\mathrm{H}} 7.51(2 \mathrm{H}, \mathrm{d}, J=8.6 \mathrm{~Hz})\right.$ and $6.87(2 \mathrm{H}, \mathrm{d}$, $J=8.6 \mathrm{~Hz})]$, an aromatic singlet proton $\left[\delta_{\mathrm{H}} 5.91(1 \mathrm{H}, \mathrm{s})\right]$, a hydrogen-bonded hydroxy group $\left[\delta_{\mathrm{H}} 14.70(\mathrm{~s})\right]$, and a 
<smiles>[R]CC([R])C</smiles>

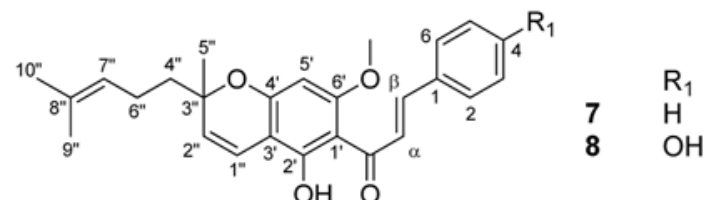<smiles>COc1ccc(/C=C/C(=O)c2c(O)cc(O)cc2O)cc1</smiles><smiles>C[C@@]12CCC[C@@H]1C(C=O)=CC[C@@H]2O</smiles><smiles>COc1cc(C=CC=CC(=O)CCc2ccc(O)cc2)ccc1O</smiles>

Fig. 1. The structures of $\mathbf{1}$ - 9 isolated from A. tsao-ko.

Table 1. NMR spectroscopic data for compound 8 (400 MHz, $\left.\mathrm{CDCl}_{3}\right)$

\begin{tabular}{|c|c|c|}
\hline Position & $\delta_{\mathrm{H}}$, mult. $(J$ in $\mathrm{Hz})$ & $\delta_{\mathrm{C}}$ \\
\hline 1 & - & 125.2 \\
\hline 2 & 7.51, d (8.6) & 130.3 \\
\hline 3 & $6.87, \mathrm{~d}(8.6)$ & 115.9 \\
\hline 4 & - & 157.6 \\
\hline 5 & $6.87, \mathrm{~d}(8.6)$ & 115.9 \\
\hline 6 & 7.51, d (8.6) & 130.3 \\
\hline $1^{\prime}$ & - & 106.0 \\
\hline $2^{\prime}$ & - & 162.5 \\
\hline 2'-OH & $14.70, \mathrm{~s}$ & - \\
\hline $3^{\prime}$ & - & 102.8 \\
\hline $4^{\prime}$ & - & 160.8 \\
\hline $5^{\prime}$ & $5.91, \mathrm{~s}$ & 91.4 \\
\hline $6^{\prime}$ & - & 162.6 \\
\hline $\mathrm{OCH}_{3}-6^{\prime}$ & $3.91, \mathrm{~s}$ & 55.7 \\
\hline $1 "$ & $6.74, \mathrm{~d}(10.0)$ & 124.2 \\
\hline $2^{\prime \prime}$ & $5.42, \mathrm{~d}(10.0)$ & 116.6 \\
\hline $3^{\prime \prime}$ & - & 80.7 \\
\hline $4 "$ & $1.81, \mathrm{~m} ; 1.69, \mathrm{~m}$ & 41.7 \\
\hline $5^{\prime \prime}$ & $1.42, \mathrm{~s}$ & 27.2 \\
\hline $6 "$ & $2.13, \mathrm{dt}(7.0,7.5)$ & 22.7 \\
\hline $7 "$ & $5.11, \mathrm{~m}$ & 123.8 \\
\hline $8^{\prime \prime}$ & - & 131.8 \\
\hline $9^{\prime \prime}$ & $1.66, \mathrm{~s}$ & 25.7 \\
\hline $10^{\prime \prime}$ & $1.58, \mathrm{~s}$ & 17.7 \\
\hline $\mathrm{CO}$ & - & 192.7 \\
\hline$\alpha$ & $7.79, \mathrm{~d}(15.7)$ & 128.4 \\
\hline$\beta$ & $7.76, d(15.7)$ & 142.3 \\
\hline
\end{tabular}

methoxy group $\left[\delta_{\mathrm{H}} 3.91(3 \mathrm{H}, \mathrm{s})\right]$. The remaining signals of the ${ }^{1} \mathrm{H}$ NMR spectrum of $\mathbf{8}$ exhibited the presence of 2methyl-2-(4-methylpent-3-enyl) $2 \mathrm{H}$-pyran ring [ $\delta_{\mathrm{H}} 6.74$

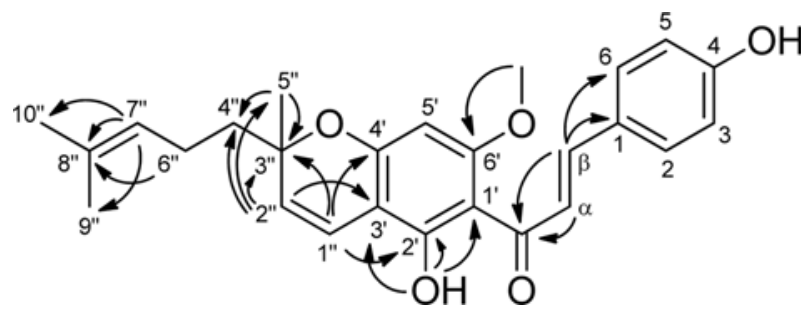

Fig. 2. Key HMBC correlations for compound 8.

$(1 \mathrm{H}, \mathrm{d}, J=10.0 \mathrm{~Hz}), 5.42(1 \mathrm{H}, \mathrm{d}, J=10.0 \mathrm{~Hz}), 5.11(1 \mathrm{H}$, $\mathrm{m}), 2.13(2 \mathrm{H}, \mathrm{dt}, J=7.0,7.5 \mathrm{~Hz}), 1.81(1 \mathrm{H}, \mathrm{m}), 1.69(1 \mathrm{H}$, $\mathrm{m})$, and 1.66, 1.58, 1.42 (each $3 \mathrm{H}, \mathrm{s})]$. The ${ }^{13} \mathrm{C}$-NMR data showed 26 carbon signals comprising a carbonyl carbon, twelve aromatic carbons, six olefinic carbons, a quaternary carbon, two methylene carbons, a methoxy carbon, and three methyl carbons (Table 1). The location of the methoxy group at C-6' position was confirmed by the HMBC correlation from the methoxy singlet at $\delta_{\mathrm{H}} 3.91$ $(3 \mathrm{H}, \mathrm{s})$ to $\mathrm{C}-6$ ' at $\delta_{\mathrm{C}} 162.6$ (Fig. 2). In addition, the HMBC correlations from $\delta_{\mathrm{H}} 6.74\left(\mathrm{H}-1^{\prime \prime}\right)$ to $\delta_{\mathrm{C}} 162.5\left(\mathrm{C}-2^{\prime}\right), 160.8$ $\left(\mathrm{C}-4^{\prime}\right)$, and 80.7 (C-3"), as well as the correlations from $\delta_{\mathrm{H}}$ 5.42 (H-2") to $\delta_{\mathrm{C}} 102.8$ (C-3'), 80.7 (C-3"), 41.7 (C-4"), and 27.2 (C-5") indicated that 2-methyl-2-(4-methylpent3-enyl) $2 \mathrm{H}$-pyran ring was attached at C-3' and C-4' (Fig. 2 ). The data of $\mathbf{8}$ are similar to those of 7 , boesenbergin $\mathrm{B}$, except for the presence of hydroxyl group at C-4. Thus, the structure of $\mathbf{8}$ was defined as (2E)-1-(5hydroxy-7-methoxy-2-methyl-2-(4-methylpent-3-enyl)2H-chromen-6-yl)-3-(4-hydroxyphenyl)prop-2-en-1-one, and named 4-hydroxyboesenbergin $\mathrm{B}$.

NO plays an important role in the inflammatory process and therefore, inhibitors of NO production may be potential anti-inflammatory agents. ${ }^{15}$ All isolates were tested for their inhibitory effects on LPS-induced NO production in RAW 264.7 cells with aminoguanidine as 
Table 2. Inhibition of LPS-induced NO production in macrophage RAW 264.7 cells of compounds $\mathbf{1}$ - 9

\begin{tabular}{cccc}
\hline \hline Compound & $\mathrm{IC}_{50}(\mu \mathrm{M})^{\mathrm{a}}$ & Compound & $\mathrm{IC}_{50}(\mu \mathrm{M})^{\mathrm{a}}$ \\
\hline $\mathbf{1}$ & $>50$ & $\mathbf{6}$ & $>50$ \\
$\mathbf{2}$ & $>50$ & $\mathbf{7}$ & 22.5 \\
$\mathbf{3}$ & $>50$ & $\mathbf{8}$ & 13.2 \\
$\mathbf{4}$ & 49.6 & $\mathbf{9}$ & 11.6 \\
$\mathbf{5}$ & 10.9 & $\mathrm{AG}^{\mathrm{b}}$ & 21.4
\end{tabular}

${ }^{\mathrm{a}}$ Results are expressed as the mean $\mathrm{IC}_{50}$ values in $\mu \mathrm{M}$ from triplicate experiments.

${ }^{\mathrm{b}}$ Aminoguanidine (AG) was used as the positive control.

the positive control ( $\mathrm{IC}_{50}$ value: $21.4 \mu \mathrm{M}$ ) (Table 2). Compounds 5, 7, and 8, which possesses a chalcone skeleton, showed significant inhibitory effects on the NO production with $\mathrm{IC}_{50}$ values ranging from 10.9 to $22.5 \mu \mathrm{M}$. Tsaokoarylone (9), a diarylheptanoid derivative, also showed considerable inhibitory activity with $\mathrm{IC}_{50}$ value of $11.6 \mu \mathrm{M}$. On the other hand, simple flavanones $(\mathbf{1}$ - 4) and a bicyclic nonane (6) showed inactive inhibitory effects $\left(\mathrm{IC}_{50}\right.$ values $\left.>50 \mu \mathrm{M}\right)$. None of the compounds showed obvious cytotoxicity at concentration of $50 \mu \mathrm{M}$ in the MTT assay (data not shown). These results indicate that three chalcones and a diarylheptanoid from $A$. tsao-ko are potential natural products for developing antiinflammatory agents.

\section{Acknowledgments}

This work was financially supported by the Research Year of Chungbuk National University in 2016.

\section{References}

(1) Tang, W.; Eisenbrand, B. Handbook of Chinese medicinal plants: Chemistry, pharmacology, toxicology; Wiley-VCH; Weinheim, 2011, p 106.

(2) Yang, X.; Küenzi, P.; Plitzko, I.; Potterat, O.; Hamburger, M. Planta Med. 2009, 75, 543-546.

(3) Lee, K. Y.; Kim, S. H.; Sung, S. H.; Kim, Y. C. Planta Med. 2008, $74,867-869$.

(4) Starkenmann, C.; Mayenzet, F.; Brauchli, R.; Wunsche, L.; Vial, C. J. Agric. Food Chem. 2007, 55, 10902-10907.

(5) Hong, S. S.; Lee, J. H.; Choi, Y. H.; Jeong, W.; Ahn, E. K.; Lym, S. H.; Oh, J. S. Tetrahedron Lett. 2015, 56, 6681-6684.

(6) Itokawa, H.; Morita, M.; Mihashi, S. Phytochemistry 1981, 20, 2503-2506.

(7) Hammami, S.; Ben Jannet, H.; Bergaoui, A.; Ciavatta, L.; Cimino, G.; Mighri, Z. Molecules. 2004, 9, 602-608.

(8) Jeon, S. H.; Chun, W.; Choi, Y. J.; Kwon, Y. S. Arch. Pharm. Res. 2008, 31, 978-982.

(9) Ibrahim, A. R.; Galal, A. M.; Ahmed, M. S.; Mossa, G. S. Chem. Pharm. Bull. 2003, 51, 203-206.

(10) McCormick, S.; Robson, K.; Bohm, B. Phytochemistry 1985, 24, 1614-1616.

(11) Moon, S. S.; Lee, J. Y.; Cho, S. C. J. Nat. Prod. 2004, 67, 889-891.

(12) Win, N. N.; Awale, S.; Esumi, H.; Tezuka, Y.; Kadota, S. J. Nat. Prod. 2007, 70, 1582-1587.

(13) Aigner, L.; Oberbauer-Hofmann, E.; Couillard-Despres, S.; Rivera, F. J.; Riepl, H.; Urmann, C.; Biendl, M. US patents, 2016, 9527860B2.

(14) Moon, S. S.; Cho, S. C.; Lee, J. Y. Bull. Korean Chem. Soc. 2005, 26, 447-450.

(15) Mariotto, S.; Suzuki, Y.; Persichini, T.; Colasanti, M.; Suzuki, H.; Cantoni, O. Curr. Med. Chem. 2007, 14, 1940-1944. 